\title{
Transaction
}

\section{Dye Fixation Process in Ink-jet Printing of Cotton Fabric by Reactive Dye}

\author{
Ryoko Yasukawa, Hiroki Higashitani, Hidekazu Yasunaga, and Hiroshi Urakawa
}

Kyoto Institute of Technology, Chemistry and Materials Technology, Sakyo-ku, Matugasaki, Kyoto 606-8585, Japan

\begin{abstract}
The dye fixation process by the contact-type dry heat fixation (CDHF) technique was investigated when cotton fabrics are dyed with a reactive dye by the ink-jet printing. The dye fixation ratio was measured as a function of the water regain of fabrics and the temperature at the fixation treatment. At first, the time dependence of the water regain and the dye fixation ratio during the treatment was observed. Although the water regain drops sharply within 2 seconds, the practical level of dyeing was achieved if the fabrics are treated with enough amount of water and with high enough temperature. The amount of water and the temperature required are $29 \mathrm{wt} \%$ and $120{ }^{\circ} \mathrm{C}$, respectively. The diffusion of dye molecules into the inside of the polymer matrix was confirmed by the model experiment using a cellophane film. In this dyeing system, the diffusion of dye molecules occurs very quickly, followed by the relatively slow dye fixation. Water molecules are very good plasticizer for the cotton fiber. The activation diffusion model may successfully describe the diffusion behavior observed in this study rather than the pore diffusion model which is often used for the explanation for the dip dyeing of cotton.
\end{abstract}

(Received 30 October, 2007 ; Accepted 19 December, 2007)

\section{Introduction}

In printing, dye fixation is carried out by heat treatments, as summarized below [1].

(1) Steaming method by saturated vapor $\left(100^{\circ} \mathrm{C}\right)$.

(2) High temperature steaming method by superheated vapor $\left(120-150{ }^{\circ} \mathrm{C}\right)$.

(3) Dry heating method by hot air (Thermofix process) $\left(150-200{ }^{\circ} \mathrm{C}\right)$.

The above-mentioned (1) and (2) techniques employ water vapor, while no water vapor is required in (3). Fewer reports have been found on the research concerning the method (3) in the printing of cotton fabric by reactive dyes.

We developed the contact-type dry heat fixation (CDHF) technique for treating cotton fabric dyed by inkjet printing [2]. The dye fixation ratio of reactive dye for cotton treated by CDHF technique in short time is higher than that of the steaming treatment even though the latter case requires the longer treatment time. The color fastness of the CDHF method is as high as that of steaming treatment.

In the report, the dye fixation mechanism in printing combined with the CDHF technique is studied in order to shed light on the feature of the technique. The authors employed an ink-jet technique in order to control the constant amount of dye liquors on fabrics during the printing. Compared to the traditional printing technique using printing color pastes, the ink-jet technique provides better reproductively of the dyeing, which is important to investigate the dyeing mechanism of printing quantitatively.

Water plays an important role of dip dyeing as a container and a carrier of dye molecules. In printing system there are lesser water molecules than in dip dyeing. The behavior and effect of water molecules in the process of the dyeing by ink-jet printing was investigated by using the system of cotton fabrics and reactive dyes. As a result the rough sketch of dyeing behavior in printing by ink-jet could be described.

\section{Experimental}

\subsection{Materials}

Broad mercerized cotton fabrics (Shikisen-sha Co. Ltd., Japan) were pre-treated as described in 2.2.1 before printing. Sodium alginate (300 cps, Nakarai Tesque Co. Ltd., Japan), sodium carbonate (Nakarai Tesque Co. Ltd., Japan) were employed for pre-treatment without further purification. To control the water regain of fabrics, lithium chloride ( $\mathrm{LiCl}$, Nakarai Tesque Co. Ltd., Japan) and potassium sulfate $\left(\mathrm{K}_{2} \mathrm{SO}_{4}\right.$, Nakarai Tesque Co. Ltd., 


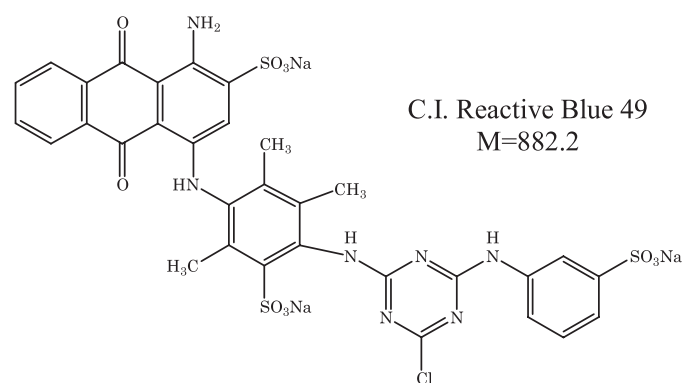

Fig. 1 Chemical structure of reactive dye used in this study.

Japan) were used. The liquid type of reactive dye, C. I. Reactive Blue 49 (Blue 49) was supplied kindly from DyStar Japan Co., Ltd. and used without further purification. Blue 49 was diluted with distilled water into the half concentration and degassed. Fig. 1 shows the chemical structure of Blue 49.

Cellophane (thickness : $40 \mu \mathrm{m}$ ) was supplied kindly from Rengo Co., Ltd. After the cellophane sample was boiled twice for 1 hour with distilled water, it was swelled for 24 hour with distilled water at room temperature. The bath ratio in weight was $350: 1$ (water : cellophane).

\subsection{Procedures}

\subsubsection{Pre-treatment of fabric}

The cotton fabrics were pre-treated in the following way. The aqueous solution for pre-treatment was contained sodium alginate and sodium carbonate. The respective concentrations of solutes were $0.5 \mathrm{wt} \%$ of sodium alginate and $3 \mathrm{wt} \%$ of sodium carbonate. The cotton fabrics were immersed into the pre-treatment solution and wrung by a mangle. The pickup rate of solution was about $84 \%$. The amount of solute (sodium carbonate and sodium alginate) per gram of cotton fabric was calculated from the concentration of pre-treatment solution and pickup rates. For example, $2.4 \times 10^{-4} \mathrm{~mol}$ of sodium carbonate was contained in 1 gram of cotton fabric, which was pre-treated by $3 \mathrm{wt} \%$ sodium carbonate solution. The pre-treated fabrics were air-dried.

\subsubsection{Ink-jet printing}

Mimaki JF-0604 (Mimaki Engineering Co. Ltd., Japan) with a piezo-electric drop-on-demand print head was employed as an ink-jet printer. The plain digital image of $4 \times 4 \mathrm{~cm}$ square prepared by a personal computer was printed at a resolution of $720 \times 720 \mathrm{dpi}$ with the aid of TxLink software (Mimaki Engineering Co. Ltd., Japan).

\subsubsection{Control of water regain in fabric}

The printed cotton fabrics were moisturized by keeping them in desiccators with saturated salt aqueous solutions for 24 hours at room temperature [3]. The water regain of all fabrics which were used for the fixation experiments was measured. The lowest water regain of printed fabrics was obtained by keeping them in a vacuum desiccator, and the highest water regain was obtained by keeping in the desiccator filled with saturated water vapor.

The amount of water in fabrics was determined by a halogen moisture analyzer HG53 (METTLER TOLEDO).

The water regain per unit mass of cotton fabric was calculated according to eq 1

$$
\text { Water regain }=W_{f} /\left(W_{0}-W_{p}\right) \times 100[\mathrm{wt} \%]
$$

Here $W_{f}$ is the mass of absorbed water in a printed cotton fabric, $W_{0}$ is the mass of the dry printed cotton fabric, and $W_{p}$ is the mass of pre-treatment reagents in the dry printed cotton fabric.

\subsubsection{Dye fixation process using the contact-type dry heat fixation (CDHF) method}

The dye fixation was carried out by CDHF technique [2] using the apparatus illustrated in Fig. 2. The dry heat device for CDHF technique consists of insulating board, heating iron plate and handgrip. The heating plate regulate within preset temperature $0.7{ }^{\circ} \mathrm{C}$ by digital temperature control system TTM-64(TOP). The fabric applied dye liquors using inkjet printer is put between dry heating plates and pressed from upper side for $60 \mathrm{~s}$ from 70 to $170{ }^{\circ} \mathrm{C}$.

\subsubsection{Calculation of dye fixation ratio}

The fabrics after fixing treatments were washed for 30 minutes with $200 \mathrm{ml}$-distilled water at room temperature. The spectra of unfixed Blue 49 in the washing solution were observed by a spectrophotometer U-2000 (HITACHI).

The Dye fixation ratio was calculated according to eq 2

Dye fixation ratio $=1-\left(A_{f} / A_{0}\right)$

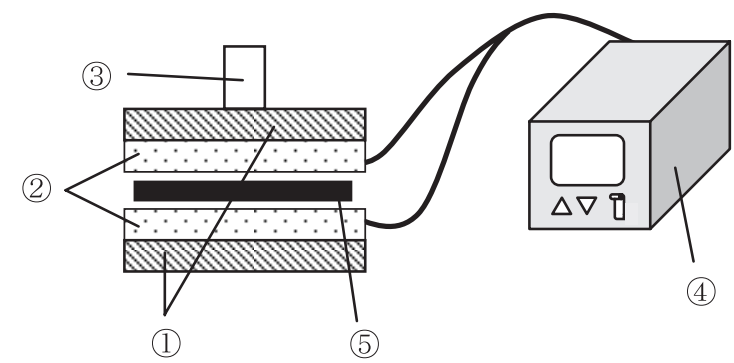

Fig. 2 Illustration of contact-type dry heat fixation apparatus. (1) : insulating board, (2): heating iron plate, (3): handgrip, (4): temperature control system, (5): fabric 
Here, $A$ means the absorbance of the washing solution at maximum absorption wavelength, $630 \mathrm{~nm}$, of Blue 49. The suffixes of $f$ and 0 denote the heat-treated fabrics and the untreated ones, respectively. $A_{0}$ is corresponding to the amount of dye which was applied by the ink-jet printer. The extraction experiments of the dye from the fabrics were also carried out to determine the dye fixation ratio more precisely. The difference between two methods was up to $10 \%$. The following discussion will be performed in terms of the dependence of the dye fixation ratio on the temperature and the heat treatment time. Accordingly an essential discussion is possible even by using the former simpler method.

\subsection{Microscope observation of cross section of ink-jet printed cellophane film}

A swollen cellophane film was immersed into 0.5 $\mathrm{wt} \%$ sodium alginate solution for 24 hours at room temperature. The water regain of the treated cellophane film was controlled to be $9.8 \mathrm{wt} \%$. Blue 49 was applied onto the cellophane film by the ink-jet printer. The cross section observations were carried out for the cellophane film heat-treated for 2 seconds at $160{ }^{\circ} \mathrm{C}$ by the CDHF technique and untreated cellophane. Before the observation the cellophane samples were embedded in epoxy resin to cut into a thin film. The cross-section was observed by digital microscope VHX-200 (KEYENCE).

\section{Results and discussions}

\subsection{Time dependence of water regain in cotton fabric and dye fixation ratio during heat treatment}

The amount of water in the fabric can not be kept constant during the heat treatment because the experimental system is open to outside. In fact, when fabric having a large amount of water is placed between two heated iron plates for the dye fixation by the CDHF technique, the vaporization of the water is often observed by the naked eye. When the fabric put in a sealed plastic bag is treated in the same way, the bag immediately expands. This also indicates that the water molecules can easily escape from the fabrics during the heat treatment. Accordingly it is important to examine the change in the amount of water in the fabrics during the treatment. The time dependence of the water regain after the start of the heat treatment was measured at several temperatures from 70 to $160{ }^{\circ} \mathrm{C}$. The result in Fig. 3 is normalized by an initial water regain. When the fabric is treated at $160{ }^{\circ} \mathrm{C}$, the water regain drops sharply from 20 to $2 \mathrm{wt} \%$ within only 2 seconds. Most water molecules disappear from the

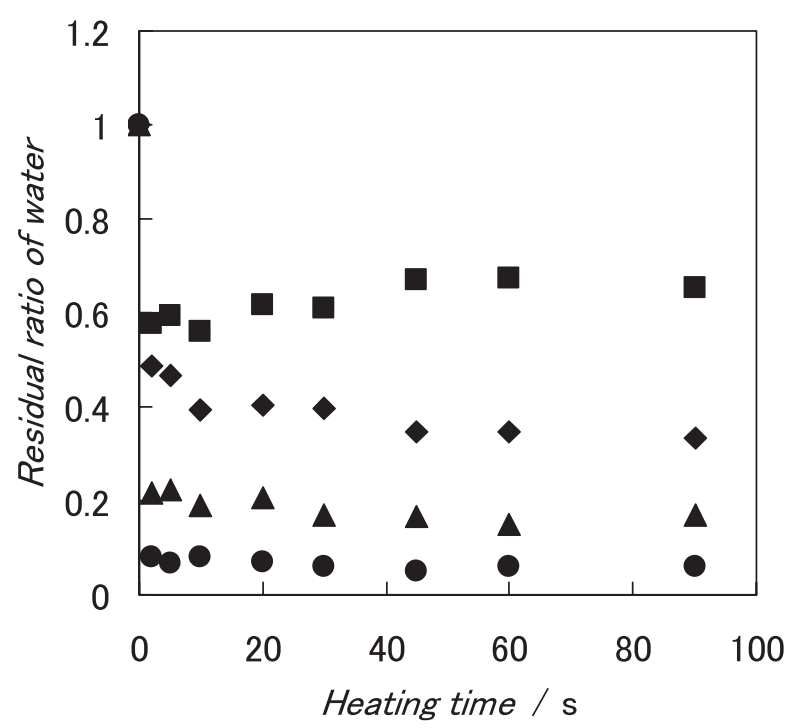

Fig. 3 The relation between heating time of the CDHF technique and normalized water regain for the pre-treated cotton fabrics. The dry heat treatment was performed at $70{ }^{\circ} \mathrm{C}(\boldsymbol{\square}), 100{ }^{\circ} \mathrm{C}(\diamond), 120{ }^{\circ} \mathrm{C}$ (A) and $160{ }^{\circ} \mathrm{C}(\mathbf{O})$.

fabric very quickly at $160{ }^{\circ} \mathrm{C}$. At the lower temperatures the sharp drops of the water regain were also observed. However, more water molecules remain in the fabrics for longer time when the treatment is carried out at the lower temperatures.

When the time dependence of the dye fixation ratio was examined at the same temperatures as in Fig. 3, the results obtained are shown in Fig. 4. The pronounced dye fixation is observed above $120{ }^{\circ} \mathrm{C}$ though water is instantaneously lost from the fabrics. The dye fixation ratio of $160{ }^{\circ} \mathrm{C}$ steeply increases until 10 seconds and afterward gradually increases. When the fabrics are treated at $120{ }^{\circ} \mathrm{C}$, the dye fixation ratio more gradually increases to the same level as $160{ }^{\circ} \mathrm{C}$. At very early stage of the heat treatment the sharp drop of the water regain and the rise of the dye fixation ratio occur at the same time. Below $100{ }^{\circ} \mathrm{C}$ the dye fixation ratios are low and less time dependences are observed compared with the higher temperatures. The latter means that the dye fixation ratio does not increase even if the fabrics are treated for longer period. The practical dyeing level requires both enough amount of water at initial stage and temperature higher than $120{ }^{\circ} \mathrm{C}$.

In order to examine whether this dye fixation occurs only at near the surface of the fiber or inside of the fiber, the model experiment was carried out by using a cellophane film instead of a cotton fiber. The crosssectional images in Fig. 5 were taken from the ink-jet printed cellophane film before (Fig. 5-a) and after (Fig. 5b) the heat treatment for 2 seconds at $160{ }^{\circ} \mathrm{C}$. As seen from Fig. 5-a, the dye, Blue 49, remains on the surface 


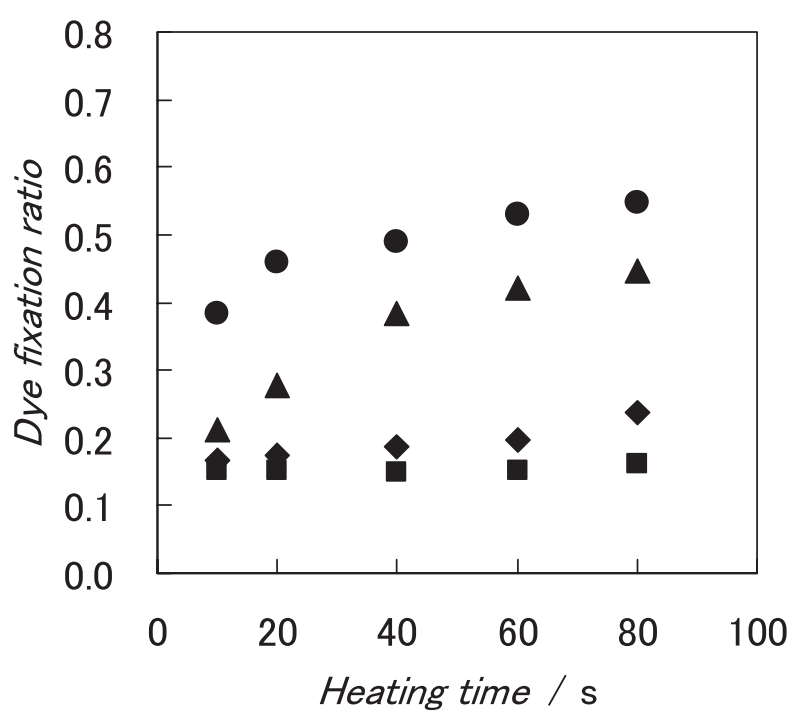

Fig. 4 The relation between heating time and dye fixation ratio of Blue 49 on cotton fabrics treated by CDHF technique at $70{ }^{\circ} \mathrm{C}(\boldsymbol{\square}), 100{ }^{\circ} \mathrm{C}(\diamond), 120$ ${ }^{\circ} \mathrm{C}(\Delta)$ and $160{ }^{\circ} \mathrm{C}(\boldsymbol{\bullet})$. The fabric contains 20 wt $\%$ of water.

cellophane before dry heating. On the other hand, a bluecolored diffusion zone clearly appears in Fig. 5-b. Dye molecules penetrate the cellophane film to a depth of about $5 \mu \mathrm{m}$. There is a strong suggestion that dye molecules can penetrate into cotton fibers by the CDHF treatment under such the condition in the same way.

\subsection{Effects of water and treatment temperature on dye fixation}

Dyeing process of reactive dyes onto cotton consists mainly of the following four steps.

(1) Adsorption of dye molecules onto the surface of cotton fibers.

(2) Diffusion of dye molecules into cotton fibers.

(3) Occupation of dye molecules at an appropriate seat in cotton fibers.

(4) Chemical bond formation between the dye molecule and cellulose.

Water molecules involve deeply the dye molecules diffusion in polymer matrix as the above step (2). There are two models for dye molecules diffusion into polymer matrix. One is a pore diffusion model. In this model a dye molecule diffuses through pores filled with water. Another is activation diffusion model related with free volume theory. Water molecules operate as a plasticizer to prompt the molecular motion of polymer chains.

The dye fixation ratios were measured for the fabrics having different water regains of 5 and $29 \mathrm{wt} \%$ at several treatment temperatures from 70 to $170{ }^{\circ} \mathrm{C}$ in order to examine the effect of water regain and treatment temperature in detail. The results are shown in Fig. 6. It is clear that the high water regain leads to high dye fixation
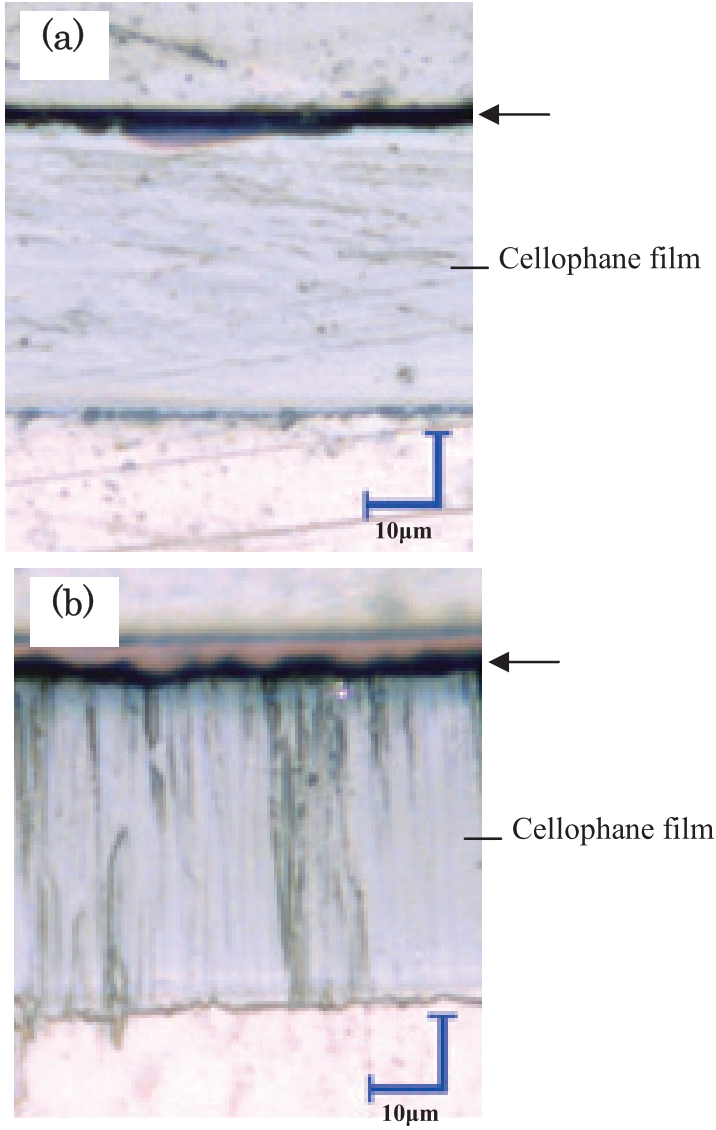

Fig. 5 Cross-section images of printed cellophane film. The part indicated by allow $(\leftarrow)$ is the printed ink layer (a): before dry heating, (b) : after dry heating.

ratio for all treatment temperatures. The dye fixation ratio for the fabric treated with $5 \mathrm{wt} \%$ of water regain is very low and does not depend on the treatment temperatures. On the other hand, the heat treatment at above $120{ }^{\circ} \mathrm{C}$ with $29 \mathrm{wt} \%$ of water regain provides the practical level of dyeing. However, the dye fixation ratio more strongly depends on the water regain in this temperature range.

The following discussion is focused on the temperature dependence of the dye fixation ratio when the cotton fabric has $29 \mathrm{wt} \%$ of water regain. The dye fixation ratio does not reach to the practical dyeing level at temperatures lower than $100{ }^{\circ} \mathrm{C}$ and increases interestingly in a staircase pattern around $120{ }^{\circ} \mathrm{C}$. Both of the increases of the water regain and the higher treatment temperature can promote the dye diffusion, followed by higher dye fixation ratio. It is difficult to judge from the data obtained in this study which is a dominant factor for the dye diffusion, temperature or water regain. The activation diffusion model may be appropriate for this system because the large temperature dependence of the dye fixation ratio is observed and there may be not enough water for filling a pore. Accordingly water 


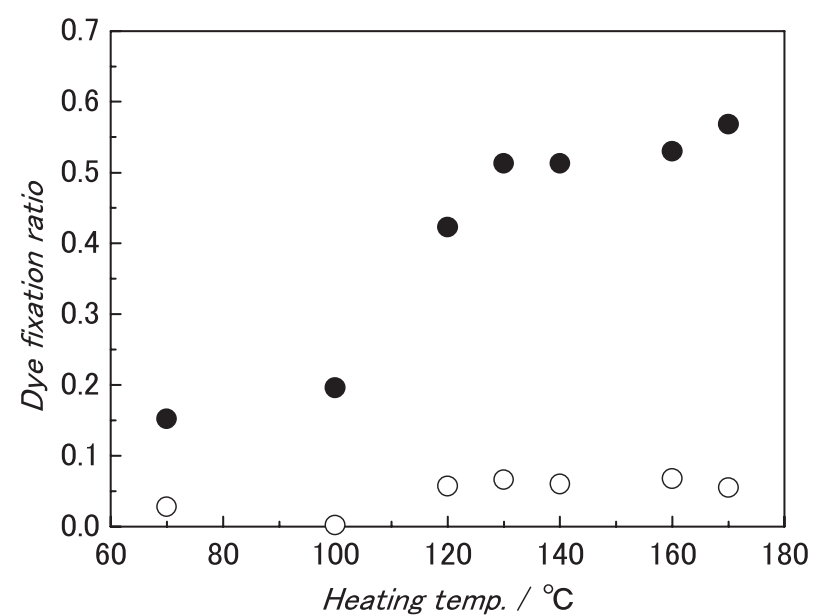

Fig. 6 The dye fixation ratio for Blue 49 plotted against the treatment temperature. Opened and closed circles denote the fabrics with $5 \mathrm{wt} \%$ and $29 \mathrm{wt} \%$ water regain, respectively. Triangles show the average value of water regain from 2 to 90 second during the treatment.

molecules act as a plasticizer to activate the molecular motion of cellulose. This activity of water is limited within less than 2 seconds from the beginning of the heat treatment. The dye molecules diffuse inside polymer matrix and settle at appropriate seats in such short time, which is followed by the slow formation of chemical bonding between a dye molecule and cellulose molecule. The gradual increase in the dye fixation ratio of the fabrics treated at 120 and $160{ }^{\circ} \mathrm{C}$ after 10 second explains this mechanism.

\section{Conclusions}

The dyeing of cotton fabrics with the reactive dye was investigated using the new techniques, which offer the quick and efficient treatment for the dye fixation.

The dyeing process with the combination of ink-jet printing and CDHF technique under the suitable conditions such as enough water and high temperature can be described as following.

(1) The dye molecules reach to the surface of cotton fibers as soon as the dye liquor is applied to the fabrics.

(2) When the high temperature is applied to the fabrics, the dye molecules at the surface start to diffuse into the inside of the fiber.

(3) The diffusion process finishes within less than 2 seconds.

(4) At the same time, the formation of the chemical bonding between the dye molecules and cellulose makes progress at a slower speed than the diffusion.

(5) Finally the dyeing level becomes to be practical within about 80 seconds.

The diffusion process may be described by the activation diffusion model. Water molecules do not secure the pathway of dye molecules but behave as a plasticizer.

\section{Acknowledgement}

The authors wish to thank DyStar Japan Co. Ltd. and Rengo Co. for their kind offers of dyestuff and cellophane film.

\section{References}

1. T. Takebe and H. Terao, "Nasen no Kiso to Jissai", Sen'i-sha, Japan, p.207 (1990).

2. R. Yasukawa, H. Yasunaga, and H. Urakawa, Sen 'i Gakkaishi, 62, 263 (2006).

3. Kobunshi Gakkai, "Zairyo to Suibun Handbook", Kyoritsu Shuppan Co. Ltd, Japan, p.239 (1968). 